\title{
Misoprostol sublingual versus vaginal para indução do parto a termo
}

\author{
Sublingual versus Vaginal Misoprostol for Labor Induction of Term Pregnancies
}

\begin{abstract}
Olimpio Barbosa de Moraes Filho슬 Rivaldo Mendes de Albuquerque ${ }^{2}$, Álvaro José Correia Pacheco ${ }^{3}$,
\end{abstract} Renata Holanda Ribeiro ${ }^{4}$, José Guilherme Cecatti ${ }^{5}$, Stefan Welkovic ${ }^{6}$

\section{RESUMO}

Objetivo: comparar efetividade e segurança de uso de comprimido sublingual de $25 \mu \mathrm{g}$ de misoprostol com o comprimido vaginal de $25 \mu \mathrm{g}$ do misoprostol na indução do parto com idade gestacional e $\geq 37$ semanas e colo uterino desfavorável. Métodos: realizou-se ensaio clínico controlado e aleatorizado, não cego, na Maternidade Monteiro de Morais (CISAMUPE), em Recife, no período de outubro de 2003 a fevereiro de 2004. Participaram do estudo 123 gestantes com idade gestacional e $\geq 37$ semanas, índice de Bishop $<8$ e fora de trabalho de parto, que apresentavam indicação para interrupção da gravidez. As gestantes aleatoriamente receberam $25 \mu \mathrm{g}$ de misoprostol sublingual ou $25 \mu \mathrm{g}$ de misoprostol vaginal a cada seis horas, até uma dose máxima de oito comprimidos $(200 \mu \mathrm{g})$. Para verificar diferenças entre os grupos foram utilizados média, desvio padrão, teste t de Student, $\chi^{2}$ para tendência e teste de Mann-Whitney. $\mathrm{O}$ valor de significação estatística adotado foi de 5\%. Resultados: não houve diferença significativa entre o número de mulheres que tiveram parto por via vaginal no grupo do misoprostol sublingual e no vaginal ( $65,5 \% \mathrm{vs} 75,8 \%, \mathrm{p}=0,22)$. Também não foi significativa a diferença do intervalo de tempo entre o início da indução e o parto ( 24 horas e 42 minutos vs 20 horas e 37 minutos, respectivamente, $\mathrm{p}=0,11$ ) entre os grupos. Os grupos, sublingual e vaginal, não mostraram também diferenças significativas em relação à síndrome de hiperestimulação $(1,7 \%$ vs 3,2\%, $\mathrm{p}=0,95)$, às incidências de mecônio $(5,2 \%$ vs 4,8\%, p=0,74), ao índice de Apgar $<7$ no quinto minuto $(3,4 \%$ vs $4,8 \%, p=0,98)$ e a outros efeitos adversos. Conclusão: o misoprostol na dose de $25 \mu \mathrm{g}$ por via sublingual apresentou a mesma efetividade e segurança quando comparado com a mesma dose vaginal para indução do parto. O misoprostol por via sublingual parece representar mais uma opção a ser considerada na indução do parto.

PALAVRAS-CHAVE: Indução do parto; Misoprostol; Gravidez normal; Parto normal; Cesárea

\section{ABSTRACT}

Purpose: to compare the effectiveness and safety of sublingual misoprostol (25 $\mu \mathrm{g})$ versus vaginal misoprostol (25 $\mu \mathrm{g})$ $\left(\right.$ Prostokos $^{\circledR}$ ) for labor induction with gestational age $\geq 37$ weeks and unripe cervices. Methods: a randomized controlled clinical trial was performed at the Maternidade Monteiro de Morais (CISAM-UPE), in Recife - PE, Brazil, from October 2003 to February 2004. One hundred and twenty-three women with gestational age $\geq 37$ weeks, Bishop score $<8$, not in labor and with medical indication for interruption of pregnancy were included in this study. The women received randomly $25 \mu \mathrm{g}$ sublingual misoprostol or $25 \mu \mathrm{g}$ vaginal misoprostol every $6 \mathrm{~h}$, not exceeding eight doses. In order to evaluate the differences between the groups, means, standard deviations, Student's t-test, $\chi^{2}$ trend and Mann-Whitney test were used. The statistical significance was considered to be 5\%. Results: there were no significant differences between the number of women with vaginal delivery in the sublingual group as compared with the vaginal group ( $65.5 \mathrm{vs} 75.8 \%, \mathrm{p}<0.22)$, or in the interval of time between the induction onset and delivery ( $24 \mathrm{~h}$ and $42 \mathrm{~min}$ vs $20 \mathrm{~h}$ and $37 \mathrm{~min}$ respectively, $\mathrm{p}=0.11)$. The two groups, sublingual and vaginal, also did not differ as to the hyperstimulation syndrome $(1.7 \mathrm{vs} 3.2 \%, \mathrm{p}=0.95)$, meconium incidence

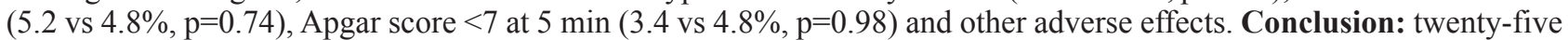
micrograms of sublingual misoprostol every six $\mathrm{h}$ presented the same effectiveness and safety as an equal vaginally administered dose of this substance. Sublingual misoprostol seems to be acceptable and is another option to be considered for labor induction.

KEYWORDS: Labor induction; Misoprostol; Normal pregnancy; Cesarean section

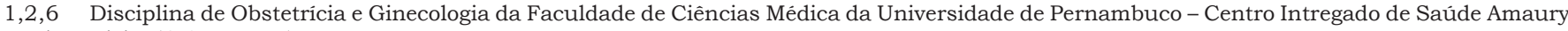
de Medeiro (CISAM-UPE)

3,4 Médico Residente - Centro Intregado de Saúde Amaury de Medeiro CISAM-UPE

5 Faculdade de Ciências Médicas da Universidade Estadual de Campinas-SP UNICAMP - Departamento de Tocoginecologia

Correspondência: Olimpio Barbosa de Moraes Filho

Rua 48, n 97, apto. 101 - Espinheiro - 52020-060 - Recife - PE - e-mail: olimpiomoraes@aol.com - Telefone: (81)3427-2112 Fax: (81)3427-3513

Recebido em: 25/11/2004 Aceito com modificações em: 10/1/2005 
Introdução

A indução do parto, quando o colo uterino não é favorável, continua sendo um dos grandes desafios da Obstetrícia moderna. De acordo com a literatura médica, o melhor agente para o amadurecimento do colo uterino e indução do parto ainda não está estabelecido. No entanto, nos últimos dez anos, a indução do parto tem se tornado um dos mais freqüentes procedimentos realizados nas maternidades dos EUA ${ }^{1}$.

No primeiro estudo publicado sobre misoprostol, para indução do parto com feto vivo, empregou-se a dose de $50 \mu \mathrm{g}$ a cada duas horas, com dose máxima de $600 \mu \mathrm{g}$, e obteve-se $73 \%$ de parto vaginal, com apenas $3,6 \%$ de síndrome de hiperestimulação ${ }^{2}$. Atualmente, o misoprostol é considerado tão ou mais eficiente que as outras prostaglandinas e mais eficaz que a ocitocina para a indução do parto com colo imaturo ${ }^{3,4}$.

A dose de $25 \mu \mathrm{g}$ por via vaginal é freqüentemente recomendada como a escolha mais prudente para indução do parto, porque está associada a menor incidência de hiperestimulação uterina ${ }^{5-7}$ e de acidose neonatal ${ }^{8}$. Em recente publicação, o American College of Obstetricians and Gynecologists $(\mathrm{ACOG})^{6}$ sugeriu que o misoprostol para indução do parto deva ser administrado pela via vaginal na dose de $25 \mu \mathrm{g}$ de quatro em quatro horas, ou de seis em seis horas.

No entanto, outros autores mostraram que, além da via vaginal, a oral e mais recentemente a sublingual são efetivas para a indução do parto, e que não há consenso na literatura sobre qual via deve ser escolhida ${ }^{9-13}$.

Alguns estudos mostraram que a dose de misoprostol necessária para induzir um parto é três a quatro vezes menor quando a droga é aplicada via vaginal em comparação com a via oral $^{11,14}$. Esses resultados podem ser explicados pela maior biodisponibilidade do misoprostol quando administrado pela via vaginal do que pela via oral ${ }^{15-17}$.

Recentemente, dois ensaios clínicos compararam a via oral e a via sublingual. O primeiro utilizou a dose de $50 \mu \mathrm{g}$ de misoprostol de quatro em quatro horas para os dois grupos, obtendo percentual maior de partos nas primeiras 24 horas $(73,8$ versus $45,7 \%)$ e menor intervalo de tempo entre o início da indução e o parto (20 versus $28,3 \mathrm{~h}$ ) nas gestantes que fizeram uso de misoprostol sublingual, sem mostrar diferenças quanto à via de parto, contratilidade uterina e resultados perinatais ${ }^{13}$. O segundo estudo comparou a dose $50 \mu \mathrm{g}$ de misoprostol por via sublingual versus $100 \mu \mathrm{g}$ de misoprostol por via oral, ambos os grupos com intervalo de 4 em 4 horas, não ultrapassando cinco doses. Os resultados deste último estudo não mostraram diferenças entre os dois grupos em relação ao número de partos vaginais dentro das primeiras 24 horas $(62,8$ versus $59 \%)$, tempo de indução $(21,8$ versus $24,1 \mathrm{~h}$ ), complicações maternas e resultados neonatais ${ }^{18}$.

Não há, no entanto, ensaio clínico publicado comparando as vias vaginal e sublingual para indução do parto. No entanto, há um estudo que comparou as concentrações plasmáticas dessas duas vias até a sexta hora, observando que a via sublingual apresenta maior biodisponibilidade e pico plasmático mais elevado do que a via vaginal. No entanto, quando o misoprostol foi empregado por via vaginal os niveis plasmáticos se mantiveram mais elevados por mais tempo ${ }^{17}$.

Recentemente, tornou-se disponível no Brasil o misoprostol em apresentação de comprimido de $25 \mu \mathrm{g}$ para uso vaginal, com indicação precisa para indução do parto e de venda exclusiva para as maternidades ${ }^{19}$. Há, entretanto, um inconveniente referente ao uso do comprimido de misoprostol por via vaginal: as mulheres preferem fazer uso do misoprostol por via oral, alegando menor desconforto e maior privacidade $^{20}$.

Há poucos estudos sobre o misoprostol via sublingual para indução do parto. Também não foi encontrado na literatura pesquisada estudo anterior que tenha realizado comparação entre esta via e a vaginal. Desta forma, a proposta deste estudo foi comparar a efetividade e a segurança destas duas vias de administração do misoprostol, na dose de $25 \mu \mathrm{g}$ de 6 em 6 horas para indução do parto a termo com feto vivo.

\section{Pacientes e Métodos}

Foi realizado estudo tipo ensaio clínico controlado e aleatorizado não cego, na Maternidade Monteiro de Morais, em Recife, PE, entre os meses de outubro de 2003 e setembro de 2004. Participaram do estudo as gestantes internadas no Centro Obstétrico ou na Enfermaria de Gestação de Alto Risco com indicação para indução do parto. Este estudo seguiu as normas brasileiras para pesquisa com seres humanos e o projeto de pesquisa foi aprovado pelo Comitê de Ética em Pesquisa da instituição.

Foram critérios de inclusão: idade gestacional e $\geq 37$ semanas, indice de Bishop $<8$, 
feto único e vivo e indicação para interrupção da gestação. Os critérios de exclusão foram: peso fetal estimado pela ultra-sonografia igual ou superior a $4.000 \mathrm{~g}$, apresentações anômalas, oligoidramnia grave (indice de líquido amniótico menor que 5), feto não reativo à cardiotocografia ou estímulo sonoro, malformações fetais, febre (temperatura $\geq 38^{\circ} \mathrm{C}$ ), cicatriz uterina e outras possiveis contraindicações para parto vaginal (placenta prévia, descolamento prematuro da placenta, herpes genital, HIV soropositivo).

As gestantes que preencheram os critérios de inclusão foram convidadas a participar do estudo. Após leitura e assinatura do termo de consentimento livre e esclarecido pelas mulheres, foi selecionado um envelope pardo selado, que foi previamente numerado em ordem seqüencial e crescente, no qual constava a ficha para a coleta de dados com o esquema de indução. Esta alocação havia sido realizada previamente de forma aleatória por computador.

As mulheres alocadas para receber misoprostol por via sublingual receberam um comprimido de $25 \mu \mathrm{g}$ de misoprostol desenvolvido especificamente para a pesquisa. As gestantes do outro grupo receberam o Prostokos ${ }^{\circledR}$ (portaria da ANVISA: 344 de 12/05/98, registro: $1.1557 .0044)$ inserido digitalmente no fundo de saco vaginal posterior. A dose foi repetida de seis em seis horas nos dois grupos, até o surgimento de pelo menos duas contrações uterinas de quarenta segundos de duração, em dez minutos, ou três contrações uterinas de trinta segundos de duração, em dez minutos (considerado trabalho de parto). A dose máxima total de misoprostol foi de $200 \mu \mathrm{g}$ (oito comprimidos). Naqueles casos em que, após seis horas da administração da oitava dose, não ocorreu trabalho de parto e o índice de Bishop continuou menor ou igual a 8 , a indução foi considerada falha. No entanto, se o índice de Bishop era superior a 8, prosseguiu-se a indução com ocitocina.

As variáveis estudadas foram: intervalo de tempo transcorrido entre a administração do primeiro comprimido e o início do trabalho de parto (latência), intervalo entre o início do trabalho de parto e o parto (duração do parto), intervalo entre o primeiro comprimido e o parto (latência + duração do parto), uso de ocitocina complementar, amniotomia, via de parto, falha de indução, indicações das cesáreas e número de doses de misoprostol.

Também foram estudadas as seguintes variáveis relacionadas aos efeitos adversos: taquissistolia, hipersistolia, sindrome de hiperestimulação, mecônio, alterações da freqüência car- diaca fetal, condições de vitalidade dos recém-nascidos (indice de Apgar ao $1^{\circ}$ e $5^{\circ}$ minuto, morte fetal, óbito neonatal, infecção neonatal e tempo de internamento do recém-nascido), efeitos colaterais maternos (náuseas, febre, calafrios) e complicações maternas (eclâmpsia, descolamento prematuro da placenta, rotura uterina e hipotonia uterina).

Foi realizada monitorização clínica de todos os partos por meio do preenchimento do partograma. A ausculta fetal e a verificação da dinâmica uterina foram realizadas no máximo a cada 60 minutos, ou a intervalos menores segundo necessidade avaliada clinicamente. As alterações da freqüência cardíaca fetal consideradas foram taquicardia ( $>160 \mathrm{bpm})$, bradicardia $(<120$ $\mathrm{bpm})$ e desacelerações tardias. Considerou-se taquissistolia a presença de seis ou mais contrações em 10 minutos, por dois periodos consecutivos de 10 minutos. Hipersistolia foi definida quando presente contração uterina de dois minutos ou mais. Considerou-se como síndrome de hiperestimulação a associação de taquissitolia e/ou hipertonia com alterações da freqüência cardiaca fetal. Quando presente taquissistolia, hipertonia ou síndrome da hiperestimulação no primeiro período do parto, a resolução por cesárea foi indicada.

Para o cálculo do tamanho da amostra, considerou-se como variável dependente principal a porcentagem de partos vaginais. Foram utilizados, como referência, os dados de estudo que relatou $73,1 \%$ de partos vaginais quando administrado misoprostol $25 \mu \mathrm{g}$ de 6 em 6 horas por via vagi$\mathrm{nal}^{21}$. Admitindo-se uma diferença mínima entre os grupos de $20 \%$ (erro alfa de $5 \%$ e um poder ( 1 $\beta$ ) de $80 \%$ ), o número total de sujeitos calculado foi de $120^{22}$. Considerando a possibilidade de perdas de seguimento de cerca de $20 \%$, procedeu-se à randomização para um total de 144 casos.

A análise estatística foi realizada com uso do programa Epi-Info 6.04. Inicialmente avaliouse a comparabilidade dos grupos, estudando-se a distribuição das variáveis de controle entre eles para testar o processo de aleatorização. A seguir, foram analizadas as distribuições de freqüências das categorias das variáveis dependentes com relação à variável independente. Para verificar a significância estatística das diferenças entre os dois grupos, utilizaram-se teste do $\chi^{2}$ para as variáveis categóricas, teste do $\chi^{2}$ para tendência para as categóricas ordenadas, teste $t$ de Student para as variáveis numéricas contínuas e teste de Mann-Whitney para as variáveis discretas ${ }^{22}$. O valor de significação estatística adotado foi de $5 \%$. 


\section{Resultados}

Inicialmente foram selecionadas 126 gestantes, mas três não concordaram em participar. Das 123 restantes, 60 foram alocadas aleatoriamente ao grupo do misoprostol sublingual e 63 ao grupo do misoprostol vaginal. No grupo do misoprostol sublingual ocorreram dois casos de suspensão do estudo (uma desistência e uma quebra de protocolo por apresentação pélvica), ao passo que no outro grupo, apenas um caso (quebra de protocolo por antecedente de cesárea anterior). Todas as exclusões ocorreram antes de se iniciar o uso do misoprostol. Assim, quando se completaram os primeiros 120 casos, 58 estavam no grupo do misoprostol sublingual e 62 no do misoprostol vaginal (Figura 1).

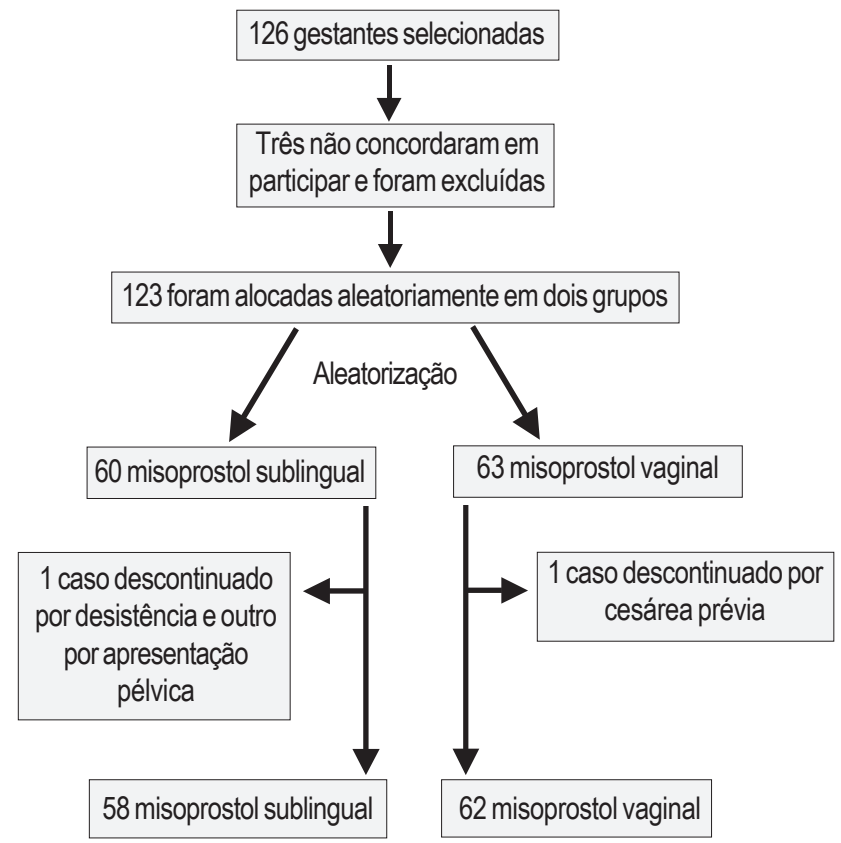

Figura 1 - Fluxo das gestantes elegíveis, incluídas, submetidas a seleção aleatorizada e as excluídas do estudo.

Os dois grupos não apresentavam diferenças significativas em relação à idade, raça, paridade, idade gestacional, peso e estatura materna, indice de Bishop, peso do recém-nascido ou indicações para indução do parto. As sindromes hipertensivas foram as principais indicações para indução do parto (Tabela 1).

Não houve diferenças significativas entre os grupos de misoprostol em relação ao período de latência ( 17 horas e 10 minutos vs 14 horas e 2 minutos, $p=0,14)$, à duração do trabalho de parto (7 horas e 32 minutos vs 6 horas e 35 minutos, $\mathrm{p}=0,18)$, ao tempo entre o início da indução e o parto ( 24 horas e 42 minutos vs 20 horas e $37 \mathrm{mi}-$ nutos, $\mathrm{p}=0,11$ ), ao uso de ocitocina complementar $(34,5$ vs $41,9 \%, p=0,55)$ e à realização de amniotomia $(31,0$ vs $17,7 \%, \mathrm{p}=0,09)$, respectivamente para a via sublingual e vaginal.

Tabela 1 - Distribuição das gestantes de acordo com algumas características sociodemográficas, estacionais e indicação da indução, segundo via de aplicação do misoprostol para indução do parto.

\begin{tabular}{lccc}
\hline Características & Sublingual & Vaginal & $\mathbf{p}$ \\
\hline Idade (média) & 24,3 & 24,0 & 0,78 \\
Cor branca (\%) & 24,1 & 29,0 & 0,54 \\
Nuliparidade (\%) & 63,8 & 69,4 & 0,42 \\
Idade gestacional (média) & 38 sem e 6 dias & 38 sem e 5 dias & 0,70 \\
Peso materno (kg, média) & 74,2 & 74,0 & 0,84 \\
Estatura materna (média) & $157 \mathrm{~cm}$ & $158 \mathrm{~cm}$ & 0,84 \\
Índice de Bishop <4 (\%) & 31 & 32 & 0,62 \\
Peso do recém-nascido (kg, média) & 3.224 & 3.190 & 0,65
\end{tabular}

Indicações para indução do parto

${ }^{*} \chi^{2}=2,11$ 0,32

Pré-eclâmpsia leve

$22,4 \quad 28,6$

Pré-eclâmpsia grave

$6,9 \quad 14,3$

Pré-eclâmpsia superposta

5,2

7,9

Hipertensão arterial crônica

10,3

7,9

Rotura prematura das membranas

17,2

20,6

Gravidez prolongada

12,1

7,9

Diabetes

12,1

6,3

Crescimento intra-uterino restrito

8,6

3,2

Oligoâmnio

5,2

3,2

Total

58

62
Embora o parto por via vaginal fosse menos freqüente no grupo do misoprostol sublingual, esta diferença não foi também significante 165,5 vs $75,8 \%, p=0,22)$. As falhas de indução do parto foram aproximadamente duas vezes mais freqüentes no grupo do misoprostol sublingual, no entanto esta diferença não foi estatisticamente significativa $(10,3$ vs 4,9\%, $p=0,43)$. Também não houve diferenças significativas em relação às indicações de cesárea entre os dois grupos (Tabela 2).

A Tabela 2 também mostra que as incidências de alterações de contratilidade uterina (taquissistolia + hipersistolia + síndrome da hiperestimulação) nos dois grupos, sublingual e vaginal, foram semelhantes $(8,5 \%$ na via sublingual vs $9,6 \%$ na via vaginal). Os dois grupos também não apresentaram diferenças significativas em relação à porcentagem de líquido amniótico com mecônio $(5,2 \%$ na via sublingual vs $4,8 \%$ na via vaginal, $p=0,74)$ e de alterações da freqüência cardíaca fetal $(5,2 \%$ na via sublingual $v s 4,8 \%$ na via vaginal, $p=0,74)$. 
Tabela 2 - Duração dos eventos relacionados ao parto, uso de ocitocina complementar, amniotomia, parto vaginal, falha de indução e indicadores das cesáreas, segundo o método de indução.

\begin{tabular}{|c|c|c|c|}
\hline & Sublingual & Vaginal & $p$ \\
\hline Latência (h, média) & 17 he $10 \mathrm{~min}$ & 14 h e 02 min & 0,14 \\
\hline Duração do parto (h, média) & 7 h e 32 min & 6 h e 35 min & 0,18 \\
\hline Latência + duração (h, média) & $24 \mathrm{~h} \mathrm{e} 42 \mathrm{~min}$ & 20 h e $37 \mathrm{~min}$ & 0,11 \\
\hline Ocitocina complementar & $20(34,5)$ & $26(41,9)$ & 0,55 \\
\hline Amniotomia & $18(31,0)$ & $11(17,7)$ & 0,09 \\
\hline Parto vaginal & $38(65,5)$ & $47(75,8)$ & 0,22 \\
\hline Falha de indução & $6(10,3)$ & $3(4,9)$ & 0,43 \\
\hline Taquissistolia & $2(3,4)$ & $3(4,8)$ & 0,93 \\
\hline Hipersistolia & $2(3,4)$ & $1(1.6)$ & 0,95 \\
\hline Síndrome da hiperestimulação & $1(1,7)$ & $2(3,2)$ & 0,95 \\
\hline Mecônio & $3(5,2)$ & $3(4,8)$ & 0,74 \\
\hline Alterações da freqüência cardíaca fetal & $3(5,2)$ & $3(4,8)$ & 0,74 \\
\hline Indicações das cesáreas & \multicolumn{2}{|c|}{${ }^{*} x^{2}=7,11$} & $p=0,62$ \\
\hline Distócias & $7(12,1)$ & $8(12,9)$ & \\
\hline Falha de indução & $6(10,4)$ & $3(4,9)$ & \\
\hline Alterações da contratilidade uterina & $4(6,9)$ & $2(3,2)$ & \\
\hline Sofrimento fetal agudo & $1(1,7)$ & $1(1,6)$ & \\
\hline Eclâmpsia & $1(1,7)$ & $1(1,6)$ & \\
\hline DPPNI & $1(1,7)$ & 0 & \\
\hline Total & 58 & 62 & \\
\hline
\end{tabular}

Teste do $\chi^{2}$ para variáveis categóricas. DPPNI = descolamento prematuro de placenta normalmente inserida.

Embora 50\% das mulheres do grupo da via sublingual tenham entrado em trabalho de parto com três comprimidos de misoprostol, contra apenas dois comprimidos no grupo do misoprostol via vaginal, não houve diferença significativa entre os dois grupos em relação à quantidade de comprimidos utilizados (Tabela 3).

Tabela 3 - Distribuição das gestantes por número de doses e dose total de misoprostol utilizada.

\begin{tabular}{lrrrrr}
\hline Doses de misoprostol & \multicolumn{2}{c}{ Sublingual } & \multicolumn{2}{c}{ Vaginal } \\
\multicolumn{1}{c}{ (dose total) } & $\mathrm{n}$ & $\%$ & $\mathrm{n}$ & $\%$ \\
\hline $1(25 \mu \mathrm{g})$ & 13 & 22,4 & 15 & 24,0 \\
$2(50 \mu \mathrm{g})$ & 7 & 12,1 & 16 & 25,0 \\
$3(75 \mu \mathrm{g})$ & 9 & 15,5 & 11 & 17,0 \\
$4(100 \mu \mathrm{g})$ & 5 & 8,6 & 9 & 14,5 \\
$5(125 \mu \mathrm{g})$ & 8 & 13,8 & 5 & 8,1 \\
$6(150 \mu \mathrm{g})$ & 4 & 6,9 & 4 & 6,5 \\
$7(175 \mu \mathrm{g})$ & 5 & 8,0 & & 0 \\
$8(200 \mu \mathrm{g})$ & 7 & 12,0 & \multicolumn{2}{c}{3} & 4,8 \\
Total & 58 & 100 & 62 & 100 \\
\hline
\end{tabular}

$\chi^{2}$ para tendência $=3,88 \quad p=0,05$.
Os indices de Apgar <7, tanto no primeiro $(17,2 \%$ na via sublingual $v$ s $12,9 \%$ na via vaginal, $\mathrm{p}=0,51)$ como no quinto minuto $(3,4 \%$ na via sublingual us 4,8 na via vaginal, $\mathrm{p}=0,98$ ), não se mostraram diferentes entre os dois grupos. A infecção neonatal ocorreu em um caso no grupo do misoprostol sublingual e em dois casos no grupo do misoprostol vaginal. Aproximadamente $90 \%$ dos recém-nascidos receberam alta nos primeiros três dias de vida em ambos os grupos (Tabela 4). Houve um caso de feto morto no grupo de misoprostol sublingual, resultante de descolamento prematuro da placenta. Esta gestante evoluiu no puerpério sem intercorrências e recebeu alta no terceiro dia pós-cesárea.

Os efeitos colaterais mais freqüentes nos dois grupos foram náuseas e vômitos, e não houve diferença entre os grupos quanto às complicações e os efeitos colaterais (Tabela 4). Não houve caso de rotura uterina nem de endometrite.

Tabela 4 - Resultados perinatais, efeitos colaterais e intercorrências maternas, segundo o método de indução.

\begin{tabular}{|c|c|c|c|c|}
\hline \multirow[t]{2}{*}{ Complicações e efeitos adversos } & \multicolumn{2}{|c|}{ Sublingual } & Vaginal & \multirow[t]{2}{*}{$p$} \\
\hline & $\mathrm{n}$ & $\%$ & n $\%$ & \\
\hline Apgar $1^{0}$ minuto $<7$ & 10 & 17,2 & 812,9 & 0,51 \\
\hline Apgar $5^{\circ}$ minuto $<7$ & 2 & 3,4 & 34,8 & 0,94 \\
\hline Infecção neonatal & 1 & 1,7 & 23,2 & 0,95 \\
\hline Internação do recém-nascido >3 dias & 6 & 8,6 & 812,7 & 0,66 \\
\hline Náuseas & 4 & 6,9 & 914,8 & 0,18 \\
\hline Vômitos & 4 & 6,9 & 34,8 & 0,93 \\
\hline Calafrios & 1 & 1,7 & 11,6 & $0,97^{*}$ \\
\hline Hipotonia uterina & 3 & 5,2 & 23,2 & 0,93 \\
\hline Eclâmpsia & 1 & 1,7 & 11,6 & 0,95 \\
\hline Descolamento prematuro da placenta & 1 & 1,7 & - & $0,24^{*}$ \\
\hline Ruptura prematura das membranas & & 1 & - & $0,89^{*}$ \\
\hline Infecção da ferida operatória & 1 & 1.7 & - & $0,89^{*}$ \\
\hline Total & & 58 & 62 & \\
\hline
\end{tabular}

${ }^{*}$ Teste exato de Fisher.

\section{Discussão}

Neste ensaio clínico não se encontraram diferenças em relação à efetividade e à segurança do uso de misoprostol quando empregado pelas vias sublingual e vaginal. No entanto, parece haver alguma sugestão de que a via vaginal seja mais efetiva que a sublingual, o que só poderia ser confirmado com aumento do tamanho amostral. Na revisão da literatura realizada, não se encontrou outro estudo 
que tenha comparado o misoprostol via sublingual com o misoprostol via vaginal para indução do parto.

O misoprostol por via vaginal apresenta-se mais efetivo que o misoprostol por via oral para a indução do parto, mesmo quando a dose da via vaginal é a metade da via oral ${ }^{10}$. Os resultados em relação à efetividade só são semelhantes quando a dose do misoprostol empregado na via oral é quatro vezes maior do que a usada na via vaginal, ou seja, $100 \mu \mathrm{g}$ versus $25 \mu \mathrm{g}^{11}$.

A via sublingual, no entanto, parece ser mais efetiva que a via oral ${ }^{12}$. É necessário que a dose do misoprostol pela via oral seja duas vezes maior do que a dose da via sublingual, para que os resultados se apresentem semelhantes em relação à efetividade, ou seja, $100 \mu \mathrm{g}$ versus $50 \mu \mathrm{g}^{18}$.

Estudos farmacológicos respaldam os resultados acima, quando mostram que a biodisponibilidade após o uso do misoprostol por via sublingual ou vaginal é maior que quando a via oral é empregada. A maior vantagem destas duas vias em relação à via oral é que, tanto na via vaginal como na via sublingual, não ocorre a primeira passagem através do figado, evitando que parte do misoprostol seja imediatamente metabolizado no sistema porta. Embora a biodisponibilidade seja maior nas primeiras seis horas e o pico máximo do misoprostol no plasma seja mais elevado na via sublingual do que na via vaginal, o nível sérico de misoprostol aplicado pela via sublingual diminui rapidamente, ao passo que na via vaginal, os níveis plasmáticos de misoprostol elevam-se mais lentamente, permanecendo por mais tempo elevados ${ }^{17}$.

Recentemente, ensaio clínico, no qual se comparou o misoprostol via sublingual versus misoprostol vaginal, mostrou que a via sublingual pode ser alternativa efetiva em relação à via vaginal para o preparo do colo antes da realização de abortamento cirúrgico ${ }^{23}$.

Assim como em outros estudos ${ }^{3,21}$, as doenças hipertensivas foram responsáveis pela maioria das indicações para indução do parto. A ruptura prematura das membranas foi a segunda indicação mais freqüente em ambos os grupos. O receio teórico de que a aplicação dos comprimidos pela via vaginal possa causar infecção ascendente não foi demonstrado no atual estudo, já que não houve nenhum caso de corioamnionite. Só houve dois casos de infecção neonatal no grupo do misoprostol via vaginal, ambos os casos de pneumonias e nenhum de sepse. No entanto, recente estudo, no qual se usou o misoprostol retal para indução do parto nos casos de ruptura prematura das membranas, com o objetivo principal de prevenir complicações infecciosas, apresentou $12,5 \%$ de corioamnionite e também de sepse neonatal ${ }^{24}$. As induções com misoprostol, em ambos os grupos, também se mostraram seguras e com baixa incidência de complicações, quando as indicações para indução foram crescimento intra-uterino restrito (CIUR), gravidez prolongada e oligoâmnio. Outro estudo também mostrou que o misoprostol pode ser usado com segurança nos casos de gravidez prolongada e CIUR ${ }^{25}$.

O presente estudo encontrou poucas falhas de indução nos dois grupos. Embora o índice de falha no grupo da via sublingual tenha sido aproximadamente duas vezes maior que no grupo da via vaginal, 10,3 versus $4,8 \%$, esta diferença não foi significativa. A falha de indução no grupo da via vaginal foi semelhante a outro estudo que utilizou a mesma via e a mesma dose, mas com intervalo de 4 horas por 24 horas no máximo ${ }^{11}$. Outro estudo mostrou índice de falha de $7,6 \%$, utilizando também a via vaginal, com a mesma dose e intervalo de administração, mas com a dose máxima de quatro comprimidos, ou seja, a metade da dose máxima do presente estudo ${ }^{21}$.

O percentual de parto vaginal foi $75,8 \%$ no grupo do misoprostol pela via vaginal, semelhante aos resultados encontrados em outros estudos ${ }^{12,21}$. Já no grupo do misoprostol sublingual, o parto vaginal ocorreu em $65,5 \%$. Este percentual de parto vaginal foi menor do que o encontrado em outros dois estudos ${ }^{13,18}$, que foi de 84 e $75,2 \%$, respectivamente, quando utilizaram dosagens duas vezes maiores $(50 \mu \mathrm{g})$, com intervalos menores (quatro em quatro horas), durante um período menor que 24 horas. Esta maior efetividade com o uso do misoprostol sublingual, em intervalos de quatro em quatro horas em comparação a intervalos de 6 em 6 horas, pode ser explicada pela queda rápida dos niveis séricos do misoprostol duas horas após sua administração ${ }^{17}$.

As alterações da contratilidade uterina e os efeitos colaterais foram semelhantes e pouco freqüentes nos dois grupos. Estes resultados são concordantes com os encontrados por outros estudos que utilizaram tanto a via vaginal como a via sublingual para indução do parto a termo ${ }^{13,18,21}$. No entanto, náuseas e vômitos parecem ser mais freqüentes quando o misoprostol é administrado pela via sublingual do que pela via vaginal na dose de $400 \mu \mathrm{g}$ com o objetivo de melhorar as condições cervicais para a realização de abortamento cirúrgico ${ }^{23}$.

O único caso de morte fetal ocorreu no grupo do misoprostol via sublingual, em gestante com hipertensão crônica que apresentou DPP. O DPP e a morte fetal provavelmente não estiveram diretamente relacionados com o uso do misoprostol, já que a gestante não se encontrava em trabalho de parto quando foi dado o diagnóstico de DPP. Os resultados do presente estudo não mostraram diferenças entre os dois grupos em relação aos re- 
sultados perinatais. As baixas incidências de infecção neonatal e índice de Apgar menor que 7 tanto no $1^{\circ}$ como no $5^{\circ}$ minuto nos dois grupos foram semelhantes a outros estudos ${ }^{5,18,21}$.

Além de os resultados do presente estudo sugerirem que a via sublingual é via segura e com efetividade semelhante à via vaginal na indução do parto a termo, há uma vantagem teórica na utilização da via sublingual, que é a sua maior aceitabilidade pelas usuárias. Recente revisão sistemática sobre o assunto concluiu, contudo, que as evidências existentes acerca do uso sublingual do misoprostol necessitam de maior número de estudos e sujeitos, para poder definitivamente concluir sobre sua efetividade e segurança ${ }^{26}$.

\section{Conflito de interesse:}

Os comprimidos de misoprostol utilizados nesta pesquisa, em suas duas formas de apresentação, foram fornecidos pelo laboratório Hebron. Não houve financiamento de outra espécie.

\section{Referências}

1. American College of Obstetricians and Gynecologists-ACOG. Committee on Obstetrics. Induction of labor. Washington, D.C.: ACOG; 1995. (Technical Bulletin, 217).

2. Margulies M, Campos Perez G, Voto LS. Misoprostol to induce labor. Lancet. 1992; 339:64.

3. Sanchez-Ramos L, Kaunitz AM, Del Valle GO, Delke I, Schroeder PA, Briones DK. Labor induction with the prostaglandin $\mathrm{E}_{1}$ methyl analogue misoprostol versus oxytocin: a randomized trial. Obstet Gynecol. 1993; 81:332-6.

4. Aquino MMA, Cecatti JG. Misoprostol versus oxytocin for labor induction in term and post-term pregnancy: randomized controlled trial. São Paulo Med J. 2003; 121:102-6.

5. Sanchez-Ramos L, Kaunitz AM, Wears RL, Delk I, Gaudier FL. Misoprostol for cervical ripening and labor induction: a meta-analysis. Obstet Gynecol. 1997; 89:633-42.

6. American College of Obstetricians and Gynecologists-ACOG. Response to Searle's drug warning on misoprostol. Washington, D.C.: ACOG; 2000. (ACOG Committee Opinion, 248).

7. Hofmeyr GJ, Gulmezoglu AM. Vaginal misoprostol for cervical ripening and induction of labour.
Cochrane Database Syst Rev. 2004; (4):CD000941.

8. Farah LA, Sanchez-Ramos L, Rosa C, et al. Randomized trial of two doses of the prostaglandin $\mathrm{E}_{1}$ analog misoprostol for labor induction. Am J Obstet Gynecol. 1997; 177:364-71.

9. Bennett KA, Butt K, Crane JM, Hutchens D, Young DC. A masked randomized comparison of oral and vaginal administration of misoprostol for labor induction. Obstet Gynecol. 1998; 92:481-6.

10.Wing DA, Ham D, Paul RH. A comparison of orally administered misoprostol with vaginally administered misoprostol for cervical ripening and labor induction. Am J Obstet Gynecol. 1999; 180:1155-60.

11. Wing DA, Park MR, Paul RH. A randomized comparison of oral and intravaginal misoprostol for labor induction. Obstet Gynecol. 2000; 95:905-8.

12. Shetty A, Danielian P, Templeton A. A comparison of oral and vaginal misoprostol tablets induction of labour at term. BJOG. 2001; 108:238-43.

13. Shetty A, Danielian P, Templeton A. Sublingual misoprostol for the induction of labor at term. Am J Obstet Gynecol. 2002; 186:72-6.

14.El-Rafaey, Rajasekar D, Abdalla M, Calder L, Templeton A. Induction of abortion with mifepristone (RU 486) and oral or vaginal misoprostol. N Engl J Med. 1995; 332:983-7.

15.Zieman M, Fong SK, Benowitz NL, Banskter D, Darney PD. Absorption kinetics of misoprostol with oral or vaginal administration. Obstet Gynecol. 1997; 90:88-92.

16.Danielsson KG, Marions L, Rodrigues A, Spur BW, Wong PY, Bygdeman M. Comparison between oral and vaginal administration of misoprostol on uterine contractility. Obstet Gynecol 1999; 93:275-80.

17.Tang OS, Schweer H, Seyberth HW, Lee WH, Ho PC. Pharmacokinetics of different routes of administration of misoprostol. Hum Reprod. 2002; 17:332-6.

18. Shetty A, Mackie L, Danielian P, Rice P, Templeton A. Sublingual compared with oral misoprostol in term labour induction: a randomized controlled trial. BJOG. 2002; 109:645-50.

19. Ministério da Saúde. Área Técnica de Saúde da Mulher. Indução do parto. In: Ministério da Saúde. Parto, aborto e puerpério: assistência humanizada à mulher. Brasília; 2001.

20.Ho PC, Ngai SW, Liu KL, Wong GCY, Lee SW. Vaginal misoprostol compared with oral misoprostol in termination of second trimester pregnancy. Obstet Gynecol. 1997; 90:735-8.

21. Moraes Filho OB. Misoprostol versus sonda Foley e ocitocina para indução do parto [tese]. Campinas: Univ. Estadual de Campinas; 2002. 
22.Pocock S. Clinical trials. A practical approach. 1st ed. New York: John Wiley \& Sons; 1983.

23. Hamoda H, Ashok PW, Flett GM, Templeton A. A randomized controlled comparison of sublingual and vaginal administration of misoprostol for cervical priming before first-trimester surgical abortion. Am J Obstet Gynecol. 2004; 190:55-9.

24. Arcanjo FCN, Alencar Júnior CA, Feitosa FEL, Amorim MMR. Uso de misoprostol retal para indução do parto em gestantes com amniorrexe prematura: ensaio clínico fase II. Rev Bras Ginecol Obstet. 2003; 25:491-9.
25.Rozemberg P, Chevret S, Sénat MV, Bretelle F, Paule Bonnal A, Bille Y. A randomized trial that compared intravaginal misoprostol and dinoprostone vaginal insert in pregnancies at high risk of fetal distress. Am J Obstet Gynecol. 2004; 191:247-53.

26.Muzonzini G, Hofmeyr GJ. Buccal or sublingual misoprostol for cervical ripening and induction of labour. Cochrane Database Syst Rev. 2004; (4):CD004221. 